\title{
Opportunistic Maintenance Policy for a System with Hidden Failures: A Multicriteria Approach Applied to an Emergency Diesel Generator
}

\author{
Cristiano A. V. Cavalcante and Rodrigo S. Lopes \\ Department of Production Engineering, School of Engineering, Centre for Technology and Geosciences, \\ Federal University of Pernambuco, 50.670-901 Recife, PE, Brazil \\ Correspondence should be addressed to Cristiano A. V. Cavalcante; cristianogesm@gmail.com
}

Received 19 April 2014; Revised 20 June 2014; Accepted 21 June 2014; Published 6 August 2014

Academic Editor: Massimo Scalia

Copyright (c) 2014 C. A. V. Cavalcante and R. S. Lopes. This is an open access article distributed under the Creative Commons Attribution License, which permits unrestricted use, distribution, and reproduction in any medium, provided the original work is properly cited.

This paper aims to develop a maintenance policy to support the maintenance decisions of an emergency system that supplies electricity when failure of the primary system occurs in a health facility. An Opportunistic maintenance policy was developed, in which failures are hidden and inspections are performed to detect the state of the system and subsystems. Failure in the system can have negative consequences for the health unit. A multicriteria model that considers cost and availability criteria and preference of the maintenance manager of the health unit was developed.

\section{Introduction}

Emergency systems that supply electricity in case of failure of primary system are present in various sectors, such as health facilities, nuclear power plants, airports, subway stations, theaters, events, such as sports games, certain companies, universities, shopping centers, military bases, water treatment and supply stations in large cities, telephony, and database stations.

In several of these cases, emergency diesel generators are used, which are triggered when a fault in the main electricity supply occurs. To continue operating as they were designed, these systems require care from the moment they are put into operation. This maintenance is required because, as the systems only operate under special circumstances, certain faults may compromise the systems' functionality when they are required to operate; therefore, tests should be performed on this equipment to determine its actual status.

The management of the maintenance of these systems is a complex task for decision makers because there are two factors that complicate decisions: (1) no one knows when system will have to be triggered because the demand for operation is unpredictable and (2) the consequences of system failures, i.e., people and the environment can be placed at risk, and losses besides economic ones can occur.

With respect to the maintenance management adopted in these systems, decision makers have to consider the characteristics inherent in the systems, in which failures are hidden and can only be perceived when starting or when inspections with functional tests are performed, and the demand of operation (triggering) is random.

This study aims to develop a maintenance policy applicable to diesel emergency generators in a health facility in Brazil. According to Brazilian law, all health units are required to maintain a source of emergency power in case of a disruption in the main supply of electricity [1].

Because the demand for its operation occurs randomly, it is essential that the equipment be operating when demanded. In this respect, one common practice is to develop a program of periodic inspections, also known as inspections per calendar or interval finding fault [2]. These inspections are conducted to ensure that the system is ready when required $[3,4]$. For the specific case of health facilities in Brazil, the inspection procedure must follow the technical standard [5], which 
advises that monthly testing be conducted on these systems to ensure that they are working properly or can receive maintenance if they exhibit defects.

These emergency systems have many components; thus, it is necessary for the maintenance policy adopted to consider the various components in the system. Wang and Pham [6] emphasize that current maintenance policies of single components are being gradually replaced by new forms of maintenance that monitor overall system performance. In this approach, instead of addressing the maintenance of isolated actions and focusing on a single component, the concept of maintenance policies for multicomponent systems aims at the coordination of maintenance activities for the system as a whole, as well as the goals that they intend to reach, namely, the minimization of costs and downtime and the maximization of reliability and availability.

The proposition of maintenance policies is a challenge for maintenance managers of emergency systems, and is a determining factor for ensuring the efficiency of management procedures that are in place in the context of maintenance. For a unit of the healthcare sector, the consequences of failure can be quite severe. A large number of lives depend on the proper functioning of these systems; in other words, people who are already debilitated have their lives at risk when these systems are not ready at the time of a primary system fails.

Therefore, it is of utmost importance to adopt models that supply decision support for keeping maintenance policies effective. Among the models for multicomponent systems is the opportunistic maintenance model, which aims to make a decision on an action to be taken in the occurrence of an opportunity.

For a system of emergency diesel generators, opportunities for maintenance actions arise over time when it is observed that one of its components has failed and this has put the entire system out of order. The decision problem is with regard to the subsystem that has not failed, that is, whether or not any action should be taken on this subsystem. If this decision is not considered, it could increase the chance of the subsystem suffering failures, shortly after the failed component is replaced. Alternatively, if maintenance is performed throughout the system whenever one of the subsystems fails, time and resources may be wasted. In view of this decision problem, a model that can describe the consequences of the decision along the dimensions of cost and availability would be extremely useful to the decision maker.

Although there are several papers in the literature concerning diesel generators and their technologies (see [7-13]), there are notably few studies that address the maintenance policy adopted in these systems, especially when applied to health units, and few studies consider more than one criterion and the preferences of the decision maker. This paper proposes a maintenance policy that considers more than one decision criterion, as applied in a system for emergency electricity supply in a health facility. For this purpose, a model was proposed, considering cost and availability in which the preferences of the decision maker are modeled by a value function.

The paper is divided into separate sections to provide details about the maintenance policy for multicomponent systems, opportunistic maintenance policies, multicriteria decision support, the proposed model, implementation and results, and, lastly, the conclusions.

\section{Maintenance Policy for Multicomponent Systems}

Emergency diesel generator systems require that the various system components be functioning at the time they are triggered. These systems are characterized as multicomponent systems, and such systems exhibit certain peculiarities regarding their maintenance policies.

Cho and Parlar [14] define multicomponent system models as models which are concerned with maintaining optimal policy for maintaining a system consisting of several different machine components or devices that may or may not depend on each other, economically, structurally, or stochastically.

In this context, these models aim to perform optimal maintenance planning for systems consisting of several components that interact with each other.

Thomas [15] classifies multicomponent systems by considering the dependence between the system components, which is divided into economic, structural, and stochastic dependence.

(i) Economic dependence, the cost of maintenance of joint components is not equal to the cost of maintenance of the individual components and can be subdivided into two classes: positive economic dependency when dependency generates some type of economy and negative economic dependency when dependency increases costs;

(ii) Stochastic dependence arises when the state of the components influences the distribution of the lifetime of other components, the interaction between existing faults or probabilistic dependence, indicating the relationship between the failed component and other components;

(iii) Structural dependence concerns cases in which a component that needs a maintenance action requires other components be disassembled or repaired.

Cho and Parlar [14] describe a second classification of models for multicomponent systems, based on stationary or dynamic aspects of planning.

(i) Stationary models assume a stable situation over time, providing static rules for maintenance, such as frequency of periodic maintenance.

(ii) Dynamic models use the information as short-term deterioration component, unexpected opportunities to generate decisions about maintenance. They have the characteristic of being dynamic over the planning horizon.

The maintenance policies for multicomponent systems have to be notably attractive, not only for providing convenience to share fixed costs among more maintenance actions but primarily for allowing an enormous number of individual 
plans to be added, making maintenance operations more efficient and simplifying the planning process. One of the policies for multicomponent systems is opportunistic maintenance. Herein, we will present, in greater detail, the policies of opportunistic maintenance because they form the basis for the development of the model proposed in our study.

\subsection{Opportunistic Maintenance Policy. Opportunistic main-} tenance can be defined as a systematic method of collection, investigation, and preplanning activities for generating a set of maintenance tasks to act on in the occurrence of an opportunity [16].

An opportunity may be related to preventive or corrective maintenance actions that result in an opportunity for action in another component, or plant shutdown.

Opportunistic maintenance is applied to multicomponent systems and is often described as a combination of shares of corrective and preventive maintenance. From a practical standpoint, the policy of opportunistic maintenance is very significant because most systems involve multiple components and often there is a certain type of dependence between components [17].

Thomas [15] notes that opportunistic maintenance is of great interest to decision makers and maintenance specialists, because opportunities arise in abundance and, without prior planning, these opportunities are being neglected.

Cui and Li [18] present a model of opportunistic maintenance for multicomponent systems. The authors considered a multicomponent cumulative damage shock model with stochastically dependent components. The likelihood of a failure of a component is associated with a threshold. So, whenever accumulative damage to a component exceeds this threshold, there is a failure. The failure of any component triggers a repair for the whole system (every component). The main contribution of the paper was to link a multi-component cumulative damage shock model with an impact analysis so as to generate a general opportunistic maintenance policy for subsystems.

Zequeira et al. [19] attempt to develop an optimal maintenance policy in conjunction with the policy of safety stock to meet demand during maintenance shutdown. They consider opportunistic maintenance to reduce overall maintenance costs.

Derigent et al. [20] propose a model of opportunistic maintenance based on fuzzy modeling considering the proximity of the components and maintainability, generating an optimal sequence of opportunistic maintenance. The economic context of maintenance and the proposed actions is considered an instance of opportunistic maintenance that supports the decision maker given the materials resources and maintenance costs involved.

Xiao-jun et al. [21] propose the opportunistic maintenance of multiunit systems, where whenever a component reaches a threshold of reliability preventive maintenance action must be performed, which creates the opportunity for preventive maintenance action on other components. The goal is to maximize the amount of preventive maintenance to be performed.
Shaomin and Longhurst [22] propose a model of opportunistic replacement based on time, considering two types of failure, type I-a failure that is resolved with minimum repair or corrective action; type II, catastrophic failure-addressed with corrective action or replacement. Their proposals are: to optimize opportunistic replacement; to extend the time warranty of the contract; and to minimize the expected maintenance costs during the life-cycle of a component.

As we have seen, there are many papers that address the problem of opportunistic maintenance, most of them doing so from an optimization perspective. On the other hand, the decision-maker may require a broader perspective to deal with this problem. Therefore, in the next section we explain some features of the Multicriteria Decision Making (MCDM) approach, which will be used to build the multicriteria model that we propose.

\section{Multicriteria Decision Support}

Those responsible for planning maintenance are faced daily with decision making involving multiple criteria; these criteria, for example, may be related to cost, availability, safety, environment, or quality. It is also observed that regarding maintenance, there are several very important issues, that in a more formal treatment, give rise to decision problems involving multiple criteria [23]. Among the main issues, we highlight the following: the definition of periodicity of maintenance, prioritizing equipment to be maintained, the allocation of teams to specific sets of tasks, classification of equipment in terms of criticality, and policy development maintenance.

These decisions share the common characteristic of having a finite set of alternatives to be evaluated from a set or coherent family of criteria. Depending on the problem, the manager may be interested in finding the best or better alternatives and ranking the alternatives from best to worst or a classification of the alternatives into predefined classes. To consider more than one criterion, decision makers can address the problem through a multicriteria method.

According to Bouyssou [24], the use of multicriteria decision support methods has the following advantages: the construction of a basis for dialogue between different agents that participate in the decision-making process, the incorporation of preferences, and the ability to evaluate the solution to address different objectives, as there may be conflict between them.

The value function consists of a systematic way to structure the decision problem and consider the trade-off between attributes; trade-offs are related to personal judgment, and the value function will help decision makers to formalize their preferences by using the structure value. The value function $v$ associates a real number $v(x)$ with each point $x$ of the consequence space, thereby reflecting the decision maker's preference with respect to the consequences and finding a value function that can be maximized and used to compare the attributes [25].

\section{Proposed Model}

The opportunistic maintenance model proposed in this study defines the maintenance actions that must be performed by 
establishing decision rules depending on the condition of the equipment at the time that inspections are conducted and detects this equipment with any component on the fail state. As maintenance decisions must result in improved equipment performance, an approach that considers more than one decision criterion is adopted so that the decision maker's preferences are respected.

To consider opportunities, the proposed model divides the system into two subsystems, 0 and 1 . The model was inspired by the calculations for opportunistic maintenance policy presented by Jorgenson et al. [26], who developed a model that optimizes availability on a jet plane. In addition to the availability function, our study models the cost function and the preferences of the decision maker using a value function.

The consideration of the subsystems, 0 and 1 , depends on the decision maker, who must select either in subsystem 1 or subsystem 0 a set of components that exhibit similar failure behavior.

Opportunities for maintenance actions arise in the course of time, whenever it is detected that one of the systems has failed, so a policy that takes into consideration what action should be taken, at an emergency, opportunistically, is extremely important. If taking action is not considered, that opportunity may be neglected, or a decision that is not good for the system may even be made.

In this sense, the model assumes that the system is composed of subsystem 0 which has an increasing failure rate and subsystem 1 which has constant failure rate. At the time of inspection, the status (failed or operating) of the system is detected.

Considering subsystems 0 and 1 , the system can fail in one of three ways: 0 fails and 1 does not fail; 1 fails and 0 does not fail; or 1 and 0 both fail.

This description implies that the decision maker has several alternatives regarding inspection points when the system's status is detected, depending on the state of the system at each point. The optimal policy for the critical limits $(n, N)$ has the following decision rules, where $n$ and $N$ are the decision variables and $T$ is the point where inspections occur to test equipment and check the system's status (failed or operational); therefore, $x, y$ and $z$ are, respectively, the operating state (1) or failed state ( 0 ) of subsystem 0 , the age of subsystem 0 , and the state of subsystem 1 .

So, the status of the system can be described by these three variables $(x, y, z)$.

(i) For $(1, T, 1)$, this means that both systems are in a good state. So, at an inspection at time $T$, do nothing, if $T<N$, and if $T=N$, replace or repair the subsystem 0 .

(ii) For $(1, T, 0)$, this means that subsystem 1 has failed, and subsystem 0 is working with age $T$. So, the recommended action is to replace or repair subsystem 1 alone, if $T<n$; otherwise, if $n<T \leq N$, replace or repair both subsystems.

(iii) For $(0, T, 0)$, this means that at the inspection on $T$, both systems have failed. This means that the recommended activity is to replace both subsystems. (iv) For $(0, T, 1)$, this means that component 0 has failed, so the recommended activity is to replace 0 alone.

To determine the value function the set of consequences is evaluated in relation to critical limits $n$ and $N$ in which each alternative has consequences in terms of cost $C(n, N)$ and availability $A(n, N)$; thus $n$ and $N$ represent a vector $a_{i}=$ $\left(n_{i}, N_{i}\right)$, where each alternative corresponds to a point in consequence space $\left(C\left(a_{i}\right) ; A\left(a_{i}\right)\right)$.

The values of cost and availability and the probability of occurrence of each possible event considered in the model are presented below together with the corresponding notations.

$p_{0}$ represents the failure of subsystem 1 , irrespective of age.

$p_{i}$ represents the failure of subsystem 0 between inspections $i-1$ and $i$, given that subsystem has not failed at $i-1$ and $p_{i} \geq p_{i-1}$.

$q_{i}$ is the failure of one subsystem or both subsystems between inspections $i-1$ and $i$, given that the subsystems are operational at $i-1$ and; $q_{i} \geq q_{i-1} . q_{i}$ determined from the relationship between $p_{0}$ and $p_{i}$ as follows

$$
q_{i}=p_{0}\left(1-p_{i}\right)+p_{i}\left(1-p_{0}\right)+p_{i} p_{0} .
$$

$f_{i}$ represents the probability of not replacing or repairing subsystem 0 until the $i$ th inspection, and it is assumed that the renewals are perfect, i.e., the system is restored to its original condition. $f_{i}$ is also the probability that the cycle ends at $i$, when the subsystem 0 is replaced or repaired. $f_{i}$ is related to $q_{i}, p_{i}$, and $p_{0}$

$$
f_{i}= \begin{cases}p_{i} \prod_{j=1}^{i-1}\left(1-p_{j}\right), & 1 \leq i \leq n-1 \\ q_{i} \prod_{j=1}^{n-1}\left(1-p_{j}\right) \prod_{j=n}^{i-1}\left(1-q_{j}\right), & n \leq i \leq N-1 \\ 1-\prod_{i=1}^{N-1} f_{i}, & i=N, \\ F_{i}=\sum_{j=1}^{i} f_{j}, & \\ F_{0}=0 . & \end{cases}
$$

4.1. Function That Describes the Attribute Availability. To determine the function that describes the attribute availability it is necessary to establish the time of each activity considered in policy. The time values considered are the following:

$$
\begin{aligned}
& t_{0}=\text { time to replace or repair subsystem } 0 \text { if not failed; } \\
& t_{00}=\text { time to replace or repair subsystem } 0 \text { upon } \\
& \text { failure; } \\
& t_{1}=\text { time to replace or repair subsystem } 1 \text { upon failure; } \\
& t_{01}=\text { time to replace or repair subsystem } 0 \text { if not failed } \\
& \text { and subsystem } 1 \text { upon failure; }
\end{aligned}
$$


$t_{01}^{\prime}=$ time to replace or repair subsystem 0 upon failure and subsystem 1 upon failure.

These time values are interesting in terms of the flexibility of the model fora given application; indeed, there may be cases in which the time to replace or repair the failed or nonfailed system will be equal or situations in which these times are different.

The limits $n$ and $N$ should be chosen such that the ratio of the long time during the cycle is maximized, that is, to return greater availability per cycle. The cycle ends when subsystem 0 is replaced or repaired.

Consider the following:

$E(X)=$ time spent to replace or repair subsystem 1 between 0 and the first failure of subsystem 0 or $n$, whichever occurs first;

$E(Y)=$ time to 0 until the first failure of subsystem 0 or end of the first cycle of failure of subsystem 1 or $N$, whichever occurs first;

$E(Z)=$ time spent to replace or repair to the end of the cycle.

The critical values $n$ and $N$ are chosen such that (3) is maximized

$$
A(n, N)=\frac{E(Y)}{E(X)+E(Y)+E(Z)} .
$$

The expected value $X$ is given by

$$
E(X)=p_{0} t_{1} \sum_{i=0}^{n-2}\left(1-F_{i}\right)\left(1-p_{i+1}\right),
$$

where

$$
\begin{gathered}
F_{i}=\sum_{j=1}^{i} f_{j}, \\
F_{0}=0 .
\end{gathered}
$$

The expected value $Y$ is given by

$$
E(Y)=\sum_{i=1}^{N-1} i f_{i}+N\left(1-\sum_{i=1}^{N-1} f_{i}\right)
$$

The expected value $Z$ is determined as follows

$$
E(Z)=\sum_{i=1}^{n-1} E Z_{i}+\sum_{i=n}^{N-1} E Z_{i}^{\prime}+\left(1-\sum_{i=1}^{N-1} f_{i}\right) t_{0}
$$

where

$$
\begin{gathered}
E\left(Z_{i}\right)=\eta_{i-1}\left(t_{01}^{\prime} p_{i} p_{0}+t_{00} p_{i}\left(1-p_{0}\right)\right), \\
\eta_{i}=\prod_{j=1}^{i}\left(1-p_{j}\right), \\
E\left(Z_{i}^{\prime}\right)=\rho_{i-1}\left(t_{01}^{\prime} p_{i} p_{0}+t_{00} p_{i}\left(1-p_{0}\right)+t_{01} p_{0}\left(1-p_{i}\right)\right), \\
\rho_{i}=\prod_{j=1}^{n-1}\left(1-p_{j}\right) \prod_{j=n}^{i}\left(1-q_{j}\right) .
\end{gathered}
$$

The values of $n$ and $N$ are maximized by (3) given the time values of each maintenance action.

4.2. Function That Describes the Attribute Cost. The model considers a system composed of two subsystems that have stochastically independent distributions of failure, but the costs are not independent when the cost to replace both subsystems is lower than the cost of replacing each subsystem separately.

Therefore, to calculate the total cost attribute the following costs are considered:

$C_{0}=$ cost to replace or repair subsystem 0 when it has not failed;

$C_{1}=$ cost to replace or repair subsystem 1 when it has failed;

$C_{01}=$ cost to replace or repair subsystem 0 when it has not failed and when subsystem 1 has failed;

$C_{00}=$ cost to replace or repair subsystem 0 when it has failed;

$C_{01}^{\prime}=$ cost to replace or repair subsystem 0 when it has failed and when subsystem 1 has failed as well.

Therefore, the cost per cycle $C(n, N)$ is described as follows: the expected number of replacements of subsystem 1 alone multiplied by the cost to replace or repair subsystem 1 when it has failed, plus the probability of replacing subsystems 0 and 1 together multiplied by the cost of replacing both subsystems, plus the probability that subsystem 0 does not fail until $N$, multiplied by the cost of replacing subsystem 0 before failure. The cost values are divided by $E(L)$, which is determined by summing $E(X), E(Y)$, and $E(Z)$ to determine the cost for the total time of the cycle.

Thus, the number of times that subsystem 1 alone is expected to be replaced, multiplied by the respective cost, is calculated as follows:

$$
p_{0} C_{1} \sum_{i=0}^{n-2}\left[\left(1-F_{i}\right)\left(1-p_{i+1}\right)\right] .
$$

The probability of replacing subsystems 0 and 1 together, multiplied by the cost of replacing both subsystems, is calculated as follows

$$
\begin{gathered}
\eta_{i}\left(\left(C_{01}^{\prime} p_{i} p_{0}\right)\right)+\left(C_{00} p_{i}\left(1-p_{0}\right)\right) \\
+\left(\rho_{i}\right)\left[\left(C_{01}^{\prime} p_{i} p_{0}\right)+\left(C_{00} p_{i}\left(1-p_{0}\right)\right)\right. \\
\left.+\left(C_{01} p_{0}\left(1-p_{i}\right)\right)\right],
\end{gathered}
$$

where

$$
\begin{gathered}
\eta_{i}=\prod_{j=1}^{i}\left(1-p_{j}\right), \\
\rho_{i}=\prod_{j=1}^{n-1}\left(1-p_{j}\right) \prod_{j=n}^{i}\left(1-q_{j}\right) .
\end{gathered}
$$


Finally, the probability that subsystem 0 does not fail until $N$, multiplied by the cost of replacing subsystem 0 before the failure, is as follows:

$$
\left[1-\sum_{i=1}^{N-1} f_{i}\right] C_{0}
$$

$E(L)$ is found by

$$
E(L)=E(X)+E(Y)+E(Z) .
$$

Based on (9), (10), (12), and (13), we can write (14) representing the cost for the total time of the cycle:

$$
\begin{aligned}
C(n, N)= & {\left[p_{0} C_{1} \sum_{i=0}^{n-2}\left[\left(1-F_{i}\right)\left(1-p_{i+1}\right)\right]\right.} \\
& +\eta_{i}\left(\left(C_{01}^{\prime} p_{i} p_{0}\right)\right)+\left(C_{00} p_{i}\left(1-p_{0}\right)\right) \\
& +\left(\rho_{i}\right)\left[\left(C_{01}^{\prime} p_{i} p_{0}\right)+\left(C_{00} p_{i}\left(1-p_{0}\right)\right)\right. \\
& \left.+\left(C_{01} p_{0}\left(1-p_{i}\right)\right)\right] \\
& \left.+\left[1-\sum_{i=1}^{N-1} f_{i}\right] C_{0}\right] \frac{1}{E(L)}
\end{aligned}
$$

Costs are minimized for certain values of $n$ and $N$ by (14).

After defining the attribute cost and availability, it is necessary to verify the independence in value between the attribute cost $C(n, N)$ and availability $A(n, N)$. According to [25], the identification of independence facilitates the determination of the value function, because it can be described as the sum of individual value functions. Therefore, the multiattribute value function can be written as

$$
U\left(C\left(a_{i}\right), A\left(a_{i}\right)\right)=\sum_{j=1}^{N} k_{j} u_{j}\left(a_{i}\right),
$$

where

$u_{j}$ is the function value of each attribute $C(n, N)$ and $A(n, N)$;

$k_{j}$ is the constant scale for each attribute;

$N$ represents the number of attributes.

Thereafter, it is necessary to verify the value functions for the attributes of $\operatorname{cost} C(n, N)$ and availability $A(n, N)$. Having verified the independence, the value functions are defined separately for each attribute.

The next step is to verify the constant of scale, which depends on the shape of the multi-attribute value function, according to [25]. For the case in which there are only two attributes, only the constant of scale for one attribute must be determined in this manner; the second attribute can be obtained by the equation $k_{1}+k_{2}=1$.

The last step is to check the consistency of the value function obtained to ensure that it actually represents the preferences of the decision maker and portrays the problem addressed.

\section{Application and Results}

As an application of the proposed model, an emergency system for electricity supply in a health facility in the city of Recife, Brazil, was studied, where an emergency diesel generator is used to supply electricity to the health unit in case of disruption from the main source. This system must be capable of supplying power to operating rooms, intensive treatment units, and emergency lights.

The health unit has a department responsible for maintaining the various pieces of equipment. The decision maker has the responsibility of determining the maintenance plan, and great attention is paid to the emergency system. The model was developed and discussed with the Decision-Maker for this system so that we could understand how well the model portrays the reality as closely as possible to elicit their preference.

For the analysis of fault data, they were divided into failure to start and failure to run, as performed in other studies of emergency diesel generator (see [10]).

Failure-to-start: Failures that occur by attempting to start the operation of the emergency diesel generator and it cannot start, or if it can start but not complete the load required to supply the health unit.

Failure-to-run: Cases in which failure does not occur when starting must maintain the required load stable over one hour of operation. If a fault occurs during this period, having started normally is considered failure to run.

To check the status of the system, periodic inspections are performed by the function test monthly (every 30 days), during which the equipment is operated for one hour. Data gathered from system failure inspections and data gathered when the system was required for operation (demanded) are shown in Table 1.

The subsystems considered for the application of the model proposed in our study are shown in Figure 1.

The system was divided into two subsystems: the first subsystem comprised the diesel engine, the electric generator, the starter, and cooling devices. Subsystem 0 has an increase in its failure rate function, over the time interval between inspections. The distribution of time to failure in this section is characterized by a conditional probability $p_{i}=i / 125$ with $i=$ $1,2, \ldots, 125$ where $p_{i}$ is the probability of this subsystem failing between actions $i$ and $i+1$ given that the system operates normally up to action $i$.

Another subsystem characterized by the subsystem 1, consists of the fuel oil device, the lubrication oil device, the control and automation device, and the output breaker. This subsystem is characterized by a constant failure rate and follows a geometric distribution with parameter $p_{0}=1 / 125$, where $p_{0}$ is the probability of this subsystem fails between two inspections.

At each inspection point which occurs every 30 days, depending on the state of each subsystem, the decision maker has several decision alternatives, and the model will find limits $n$ and $N$ that are related to the determination of these intervals and define the decision rules that must be followed by the decision maker, and these decision rules are shown in Figure 2. 


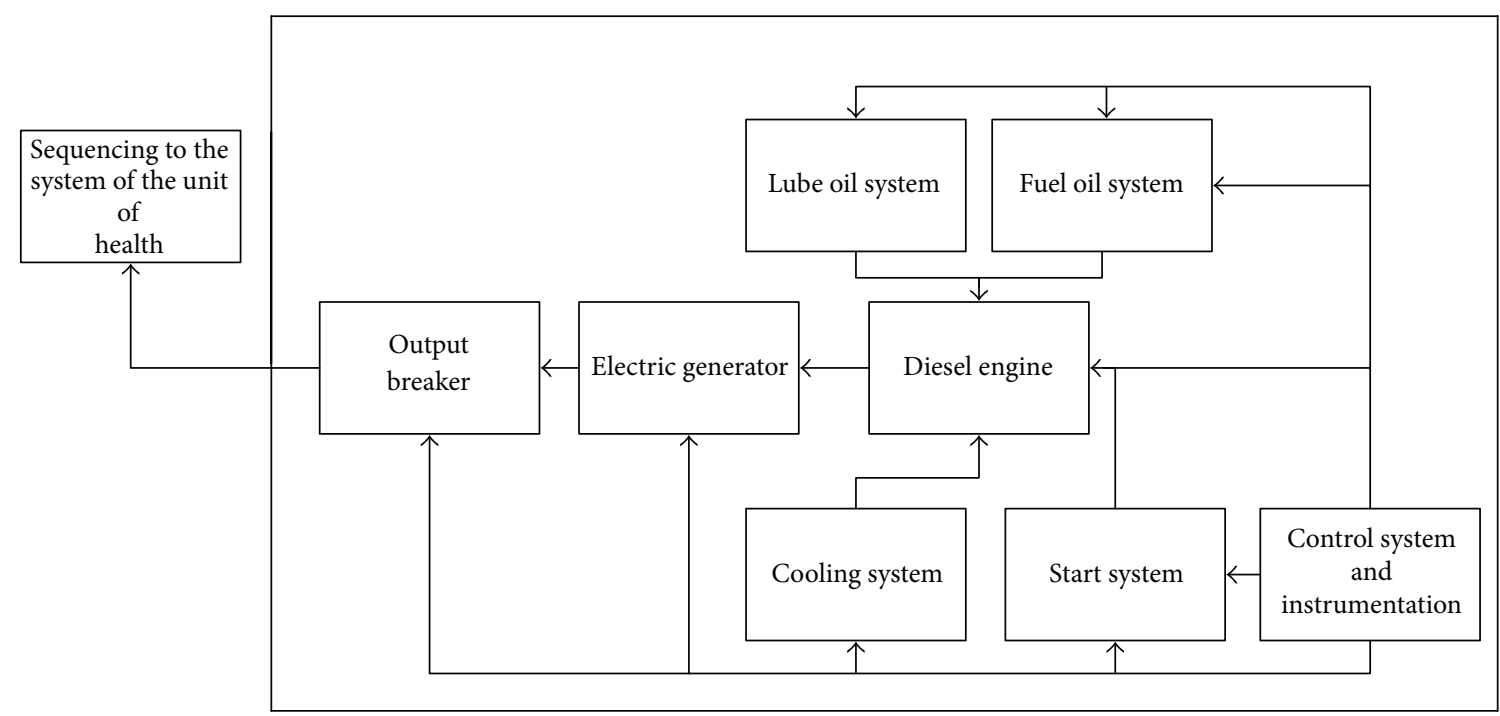

FIGURE 1: Subsystems of emergency diesel generator studied.

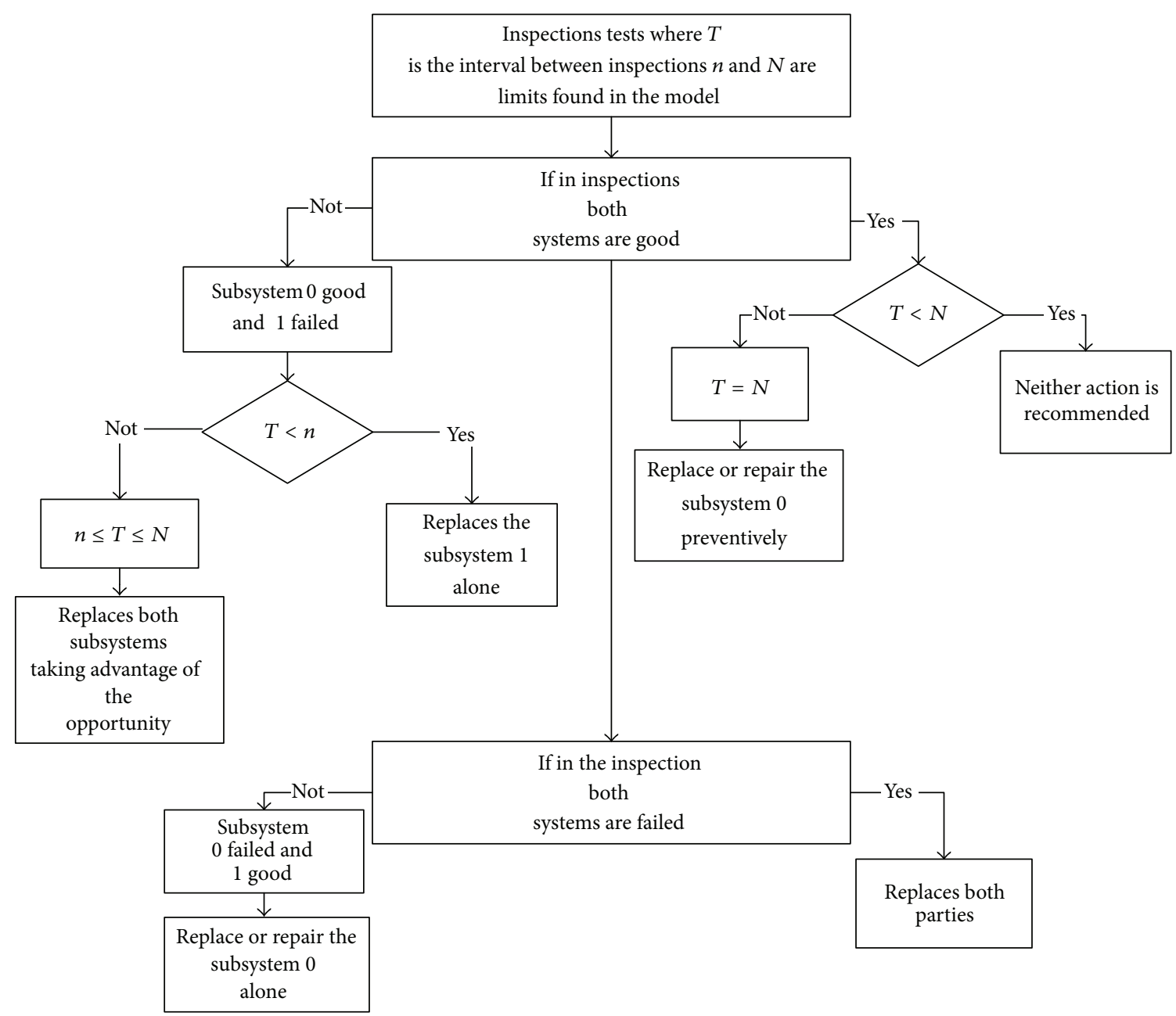

Figure 2: Decision rules. 
TABLE 1: Data regarding equipment failure.

\begin{tabular}{|c|c|c|c|c|c|c|}
\hline \multicolumn{4}{|c|}{ Inspections } & \multicolumn{3}{|c|}{ Demand per year } \\
\hline Year & Number of inspections performed/year & Failure to start & Failure to run & Demand per year & Failure to start & Failure to run \\
\hline 2000 & 12 & 3 & 1 & 42 & 7 & 2 \\
\hline 2001 & 12 & 2 & 0 & 31 & 5 & 0 \\
\hline 2002 & 12 & 1 & 0 & 28 & 2 & 0 \\
\hline 2003 & 12 & 3 & 1 & 24 & 4 & 0 \\
\hline 2004 & 12 & 4 & 2 & 20 & 3 & 1 \\
\hline 2005 & 12 & 2 & 0 & 22 & 6 & 1 \\
\hline 2006 & 12 & 1 & 0 & 26 & 4 & 0 \\
\hline 2007 & 12 & 3 & 1 & 33 & 2 & 0 \\
\hline 2008 & 12 & 2 & 1 & 29 & 4 & 1 \\
\hline 2009 & 12 & 2 & 0 & 31 & 6 & 0 \\
\hline 2010 & 12 & 1 & 1 & 36 & 3 & 1 \\
\hline 2011 & 12 & 3 & 0 & 33 & 4 & 0 \\
\hline 2012 & 12 & 2 & 1 & 37 & 6 & 1 \\
\hline 2013 & 12 & 2 & 0 & 41 & 4 & 1 \\
\hline Total & 168 & 31 & 8 & 433 & 60 & 8 \\
\hline
\end{tabular}

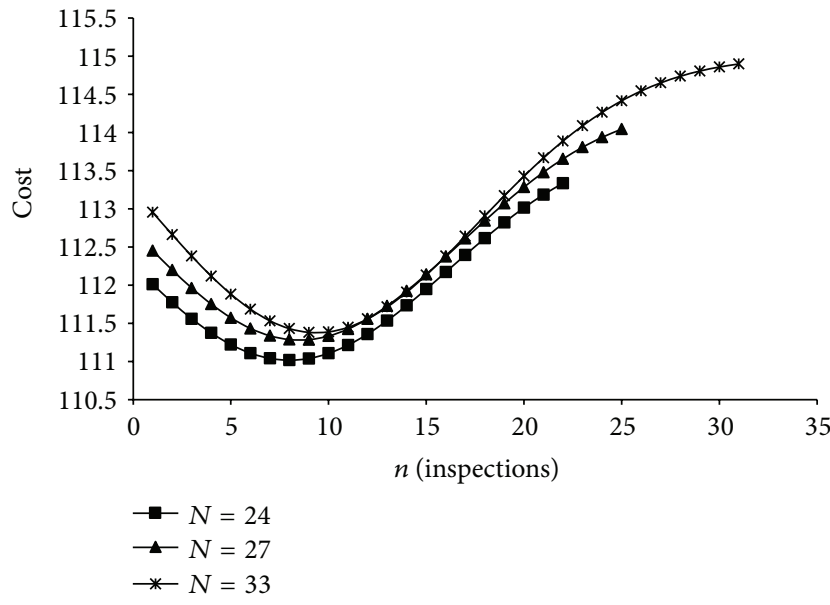

FIgURE 3: Behavior of the attribute cost.

So that the decision maker can better understand the behaviour of the different criteria, we show the decisionmaker graphical displays of the cost and availability for different combinations of $N$ and $n$.

The behavior of the cost function shows that for every $N$ there are different cost values depend on the $n$ to consider opportunities, as shown in Figure 3. As well for availability in Figure 4.

After the decision maker understands the different behaviour of the different criteria for the distinct combinations of $n$ and $N$, it is possible to find the value function of each attribute.

The decision maker should be able to see that there is a conflict between the attributes of cost and availability. In addition, he should be able to understand how this conflict is associated with the decision rules, before the preference function for each attribute is elicited. Doing so in this way, we

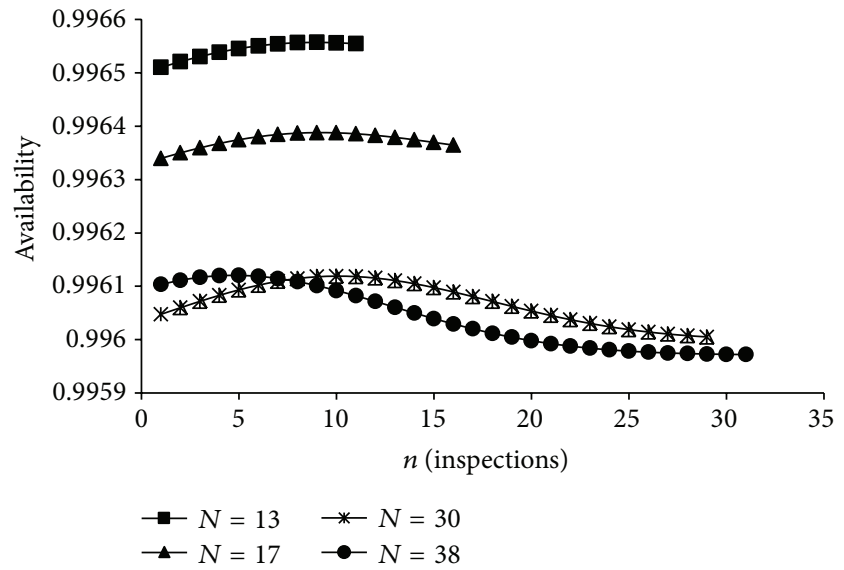

FIGURE 4: Behavior of the attribute availability.

can ensure that the decision maker can actually demonstrate his preference. After this data regarding the time and cost of implementing the model were collected from the database of the health unit and are shown in Table 2.

5.1. Determining the Space of Consequences and the Value Function. For the application of the value function and to find the space of consequences, various combinations of $n$ and $N$ were considered, yielding different cost $C(n, N)$ and availability $A(n, N)$ values, where $n$ was varied from 2 to 125 and $N$ was varied from 3 to 125 , respecting the restriction $N>n$.

To determine the space of consequences, we found the maximum and minimum limits for each attribute. For the cost attribute, the maximum value for the cost was $C(3,3)$ resulting in a cost of 309.15 . The minimum value for the cost was obtained at $C(15,23)$ resulting in a cost of 111.47. For the availability attribute, the maximum value found was 
TABLE 2: Input values for the model.

\begin{tabular}{ll}
\hline Symbols & Values for times \\
\hline$t_{1}$ & 8 hours to replace or repair subsystem 1 when it has failed \\
$t_{0}$ & 14 hours to replace or repair subsystem 0 when it has not failed \\
$t_{01}$ & 18 hours to replace or repair subsystem 0 when it has not failed and when subsystem 1 has failed \\
$t_{00}$ & 39 hours to replace or repair subsystem 0 when it has failed \\
$t_{01}^{\prime}$ & 50 hours to replace or repair subsystem 0 when it has failed and when subsystem 1 has failed \\
\hline Symbols & Values of costs \\
\hline$C_{1}$ & 800 the cost to replace or repair subsystem 1 when it has failed \\
$C_{0}$ & 900 the cost to replace or repair subsystem 0 when it has not failed \\
$C_{01}$ & 1110 the cost to replace or repair subsystem 0 when it has not failed and when subsystem1 has failed \\
$C_{00}$ & 1500 the cost to replace or repair subsystem 0 when it has failed \\
$C_{01}^{\prime}$ & 1800 the cost to replace or repair subsystem 0 when it has failed and when subsystem 1 has failed \\
\hline
\end{tabular}

TABLE 3: Value function for the attribute Cost.

\begin{tabular}{lccc}
\hline Attribute & Value function & \multicolumn{2}{c}{ Parameters } \\
\hline Cost & $u_{1}=X_{1} \exp \left(-Y_{1} C\left(a_{i}\right)\right)$ & $X_{1}=3.019$ & $Y_{1}=0.01$ \\
\hline
\end{tabular}

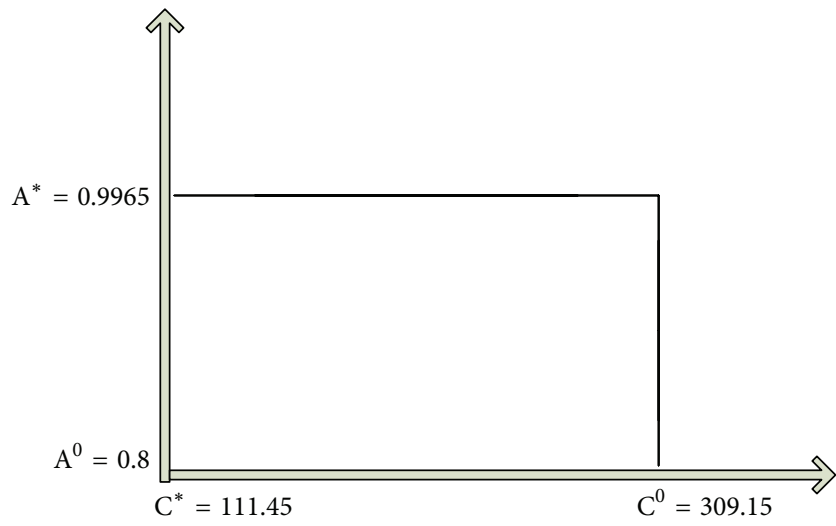

FIGURE 5: Space of consequence.

$A(12,13)$, resulting in an availability of 0,9965 , and the minimum value accepted by the decision maker for availability was 0.8 . In other words, the decision maker did not accept an availability lower than 0.8 . The space of consequence found is shown in Figure 5.

Regarding the determination of the value function with respect to the cost is represented by $u_{1}$, where $C\left(a_{i}\right)$ corresponds to the decision maker's preference related to the cost for each vector $a_{i}\left(n_{i}, N_{i}\right)$. This value function $u_{1}$ is assumed to be 1 when the cost is minimal $C(15,23)=111.45$ and assumes the value of 0 when cost is maximum $C(3,3)=309.15$. The function $u_{1}$ is an exponential function, as shown in Table 3 .

The value function for availability is represented by $u_{2}$ where $A\left(a_{i}\right)$ represents the value for availability for each vector $a_{i}$. When the availability is maximum 0.9965 , the $u_{2}$ assumes 1 , This occurs when $a_{i}=(12,13) ; u_{2}$ is assumed to be 0 when availability is the minimal 0.8 . Function $u_{2}$ that represents the decision-maker's preference related to availability is a logistic function, as shown in Table 4 .
TABLE 4: Value function for the attribute Availability.

\begin{tabular}{lccc}
\hline Attribute & Value function & \multicolumn{2}{c}{ Parameters } \\
\hline Availability & $u_{2}=X_{2} \exp \left(-Y_{2} / A\left(a_{i}\right)\right)$ & $X_{2}=6.69$ & $Y_{2}=1.8989$
\end{tabular}

We first need to find the constant of scale to determine the global value function. With the decision maker constants of scale were found 0.2 for the attribute cost and 0.8 for the attribute availability. After the determination of the scale constants and of the value function for each attribute, it is possible to assess the global value function representing the two attributes. The global value function that represents the decision maker's preference is given by

$$
u g(n, N)=k_{1} u_{1}+k_{2} u_{2}
$$

By determining the global value function and maximizing this function we obtained the optimal policy according to the decision maker's preference, that is, $n=5$ and $N=20$, which corresponds to a global value of $u g(5,20)=0.995$. We can see that the best policy for the decision-maker achieves a very good performance in both criteria, thereby giving a return in terms of cost $C(5,20)=111.01$ and availability $A(5,20)=0.9960$. The graph of the global value function is given in Figure 6.

It can be observed that the global value function achieved better results compared with the results of individual optimization of each attribute.

The multicriteria model estabilished based on the value function resulted in a global value function maximum at $n=5$ and $N=20$ corresponding to a cost $C(5,20)=$ 111.01 and availability $A(5,20)=0.9960$ and thus resulting in high availability at a moderate cost compared with the results obtained for each attribute individually.

The results obtained facilitate the implementation of the policy establishing decision rules for the actions to be taken, depending on whether subsystem 0 or 1 has failed in the inspection $T$ (performed monthly) with the limits $n=5$ and $N=20$. 


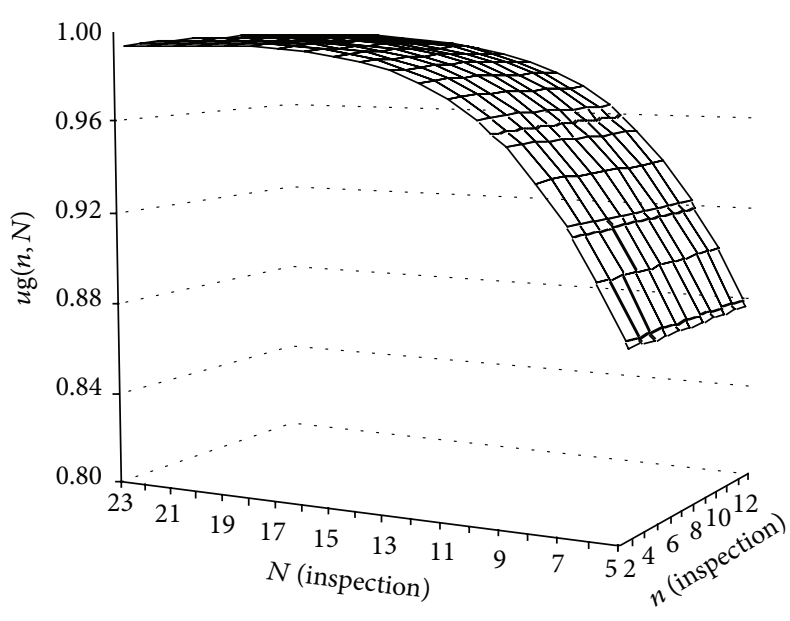

FIgURE 6: Global value function $u g(n, N)$.

\section{Conclusions}

In this study, a maintenance policy for an emergency system composed of an emergency diesel generator with hidden failures, in which failure can pose risks to the people who are being attended to or are in the health unit, was developed. The quantification and characterization of the attributes through a value function provided results consistent with the preferences of the decision maker, who does not want any failure to affect clients. Results were measured along the dimension of availability, where availability should be obtained at an acceptable cost because the health unit aims to earn profits, and the budget for the actions associated with equipment maintenance is limited.

Maintenance policies for emergency systems are of great interest to decision makers and maintenance specialists because these systems are present in various sectors and, without prior planning, decisions that consider the entire system become notably difficult. Therefore, it is important to create methodologies, methods, techniques, or models to allow for decisions related to these systems to be taken.

An opportunistic policy specified by a multicriteria perspective not only yields better results by exploiting new opportunities, but also achieves better results due to the broader perspective provided to the decision maker. In practice, the maintenance manager not only needs to know what to do but also needs to be sure that anything to be done must provide a cost reduction or enhanced equipment performance. Therefore, the model proposed in this paper provides the maintenance manager with tools to make better decisions to achieve these objectives.

\section{Conflict of Interests}

The authors declare that there is no conflict of interests regarding the publication of this paper.

\section{Acknowledgments}

This work was partially supported by CNPq (Brazilian Research Council) and FACEPE (Foundation of Science and Technology of Pernambuco State).

\section{References}

[1] Ministério da Saúde, Normas Para Projetos Físicos de Estabelecimentos Assistenciais de Saúde, Ministério da Saúde, Brasília, Brazil, 1995.

[2] T. Tang, D. Lin, D. Banjevic, and A. K. S. Jardine, "Availability of a system subject to hidden failure inspected at constant intervals with non-negligible downtime due to inspection and downtime due to repair/replacement," Journal of Statistical Planning and Inference, vol. 143, no. 1, pp. 176-185, 2013.

[3] M. D. Berrade, C. A. V. Cavalcante, and P. A. Scarf, "Maintenance scheduling of a protection system subject to imperfect inspection and replacement," European Journal of Operational Research, vol. 218, pp. 716-725, 2012.

[4] C. A. V. Cavalcante, P. A. Scarf, and A. T. de Almeida, "A study of a two-phase inspection policy for a preparedness system with a defective state and heterogeneous lifetime," Reliability Engineering \& Systems Safety, vol. 96, pp. 627-635, 2011.

[5] Associação Brasileira de Normas Técnicas, NBR 13534: Instalações Elétricas de baixa tensão : Requisitos para instalação em estabelecimentos assistenciais de saúde, Associação Brasileira de Normas Técnicas, Rio de Janeiro, Brazil, 1995.

[6] H. Wang and H. Pham, Reliability and Optimal Maintenance, Springer Series in Reliability Engineering, Springer, New York, NY, USA, 2006.

[7] W. E. Vesely, S. P. Uryasev, and P. K. Samanta, "Failure of emergency diesel generators: a population analysis using empirical Bayes methods," Reliability Engineering and System Safety, vol. 46, pp. 221-229, 1994.

[8] S. Y. Choi, J. H. Lee, and J.-E. Yang, "An analysis for risk impact of emergency diesel generator on modified surveillance test interval by considering failure due to demand stress," Nuclear Engineering and Design, vol. 240, pp. 3577-3581, 2010.

[9] M. Zubair and Z. Zhijian, "Reliability Data Update Method (RDUM) based on living PSA for emergency diesel generator of Daya Bay nuclear power plant," Safety Science, vol. 59, pp. 72-77, 2013.

[10] D. J. Winfield, "Long term reliability analysis of standby diesel generators," Reliability Engineering \& System Safety, vol. 21, no. 4, pp. 293-308, 1988.

[11] S. Hossain and N. Saiful Bari, "Waste heat recovery from the exhaust of a diesel generator using rankine cycle," Energy Conversion and Management, vol. 75, pp. 141-151, 2013.

[12] S. Lyshevski, "Analysis of auxiliary power systems with conventional synchronous generators," Energy Conversion and Management, vol. 41, pp. 1379-1387, 2000.

[13] M. S. Ismail, M. Moghavvemi, and T. M. I. Mahlia, “Technoeconomic analysis of an optimized photovoltaic and diesel generator hybrid power system for remote houses in a tropical climate," Energy Conversion and Management, vol. 69, pp. 163173,2013

[14] I. D. Cho and M. Parlar, "A Survey on maintenance models for multi-unit systems," European Journal of Operational Research, vol. 51, pp. 1-23, 1991.

[15] 1. Thomas, "A survey of maintenance and replacement models for maintainability and reliability of multi-item systems," Reliability Engineering, vol. 16, pp. 297-309, 1986.

[16] J. A. Day and L. George, "Opportunistic replacement of fusion power system parts," in Proceedings of the Reliability and Maintainability Symposium, vol. 22, pp. 16-27, 1982. 
[17] R. C. Vergin and M. Scriabin, "Maintenance scheduling for multicomponent equipment," AIIE Transactions, vol. 9, pp. 297305, 1977.

[18] L. Cui and H. Li, "Opportunistic maintenance for multi-component shock models," Mathematical Methods of Operations Research, vol. 63, no. 3, pp. 493-511, 2006.

[19] R. Zequeira, J. Valdes, and C. Berenguer, "Optimal buffer inventory and opportunistic preventive maintenance under random production capacity availability," International Journal of Production Economics, vol. 111, pp. 686-696, 2008.

[20] T. Derigent, E. Thomas, E. Levrat, and B. Iung, “Opportunistic maintenance based on fuzzy modelling of component proximity," CIRP Annals-Manufacturing Technology, vol. 58, no. 1, pp. 29-32, 2009.

[21] Z. Xiao-Jun, L. Zhi-Qiang, X. Li-Feng, and L. Jay, “Opportunistic preventive maintenance optimization for multi-unit series systems with combing multi-preventive maintenance techniques," Journal of Shanghai Jiaotong University (Science), vol. 15, pp. 513-518, 2010.

[22] W. Shaomin and P. Longhurst, "Optimising age-replacement and extended non-renewing warranty policies in lifecycle costing," International Journal of Production Economics, vol. 130, pp. 262-267, 2011.

[23] C. A. V. Cavalcante, P. R. J. Ferreira, and A. T. Almeida, "A preventive maintenance decision model based on multicriteria method PROMETHEE II integrated with Bayesian approach," IMA Journal of Management Mathematics, vol. 21, pp. 333-348, 2010.

[24] D. Bouyssou, "Decision Multicritére ou Aide Multicritére," Bulletin du Groupe de Travail europénn; Aide multicritére à la decision, vol. 2, no. 2, p. 2, 1993.

[25] R. L. Kenney and H. Raiffa, Decisions with Multiple Objectives: Preferences and Value Tradeoffs, Cambridge University Press, Cambridge, UK, 1976.

[26] D. Jorgenson, J. McCall, and R. Radner, "Optimal Maintenance of Stochastically Failing Equipment," United States Air Force Project RAND, 1966. 


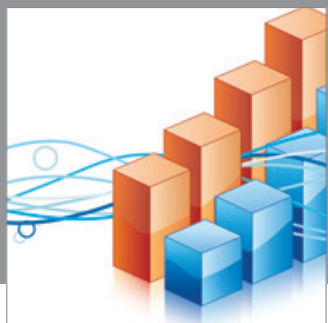

Advances in

Operations Research

mansans

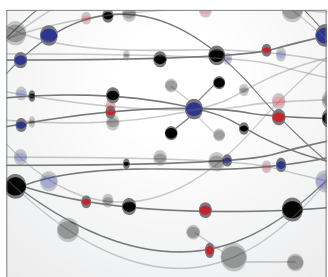

The Scientific World Journal
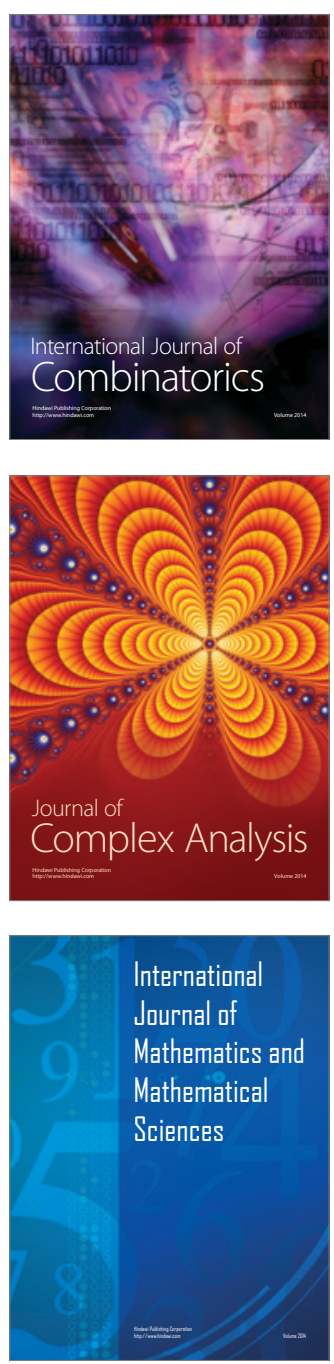
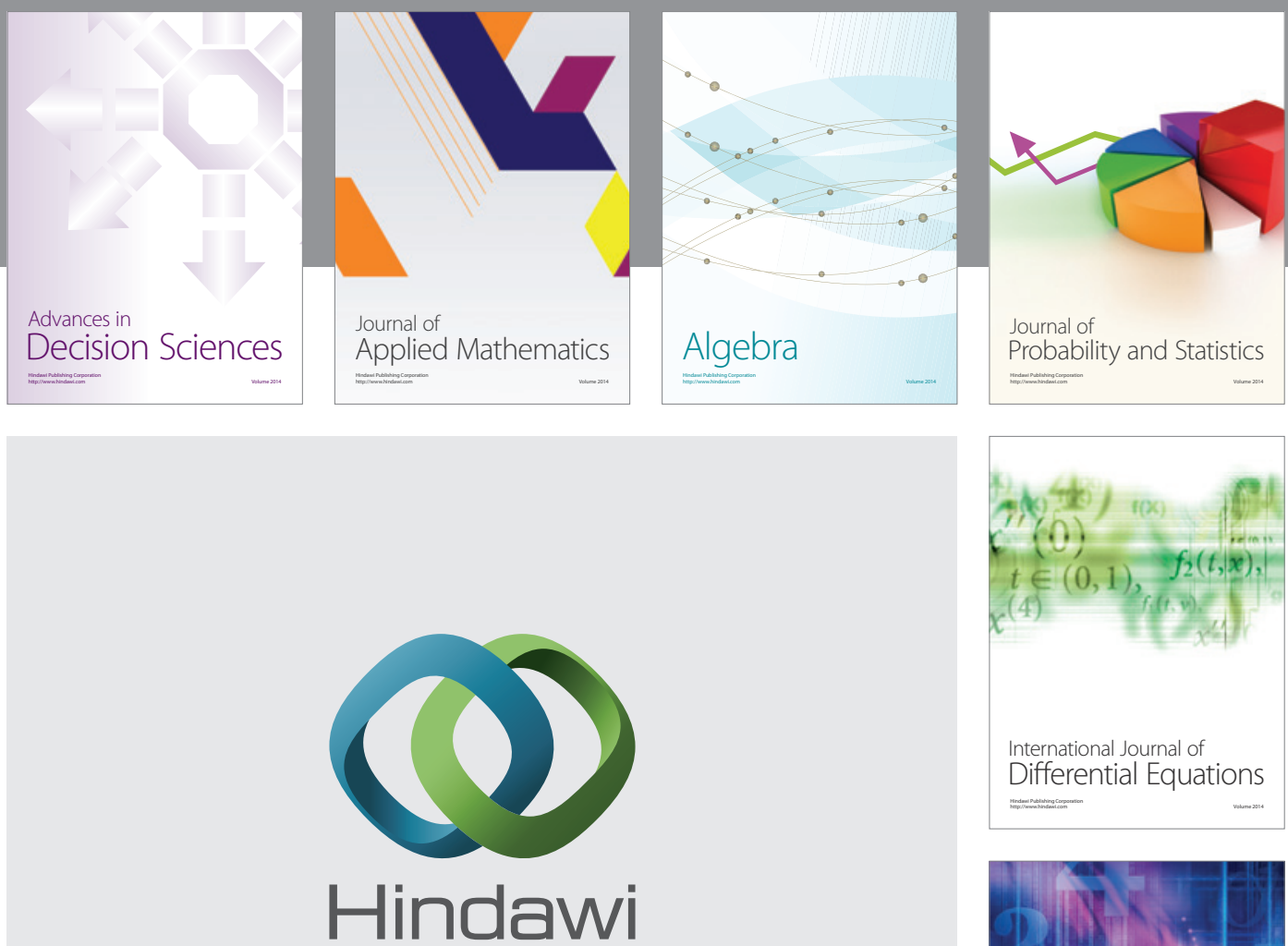

Submit your manuscripts at http://www.hindawi.com
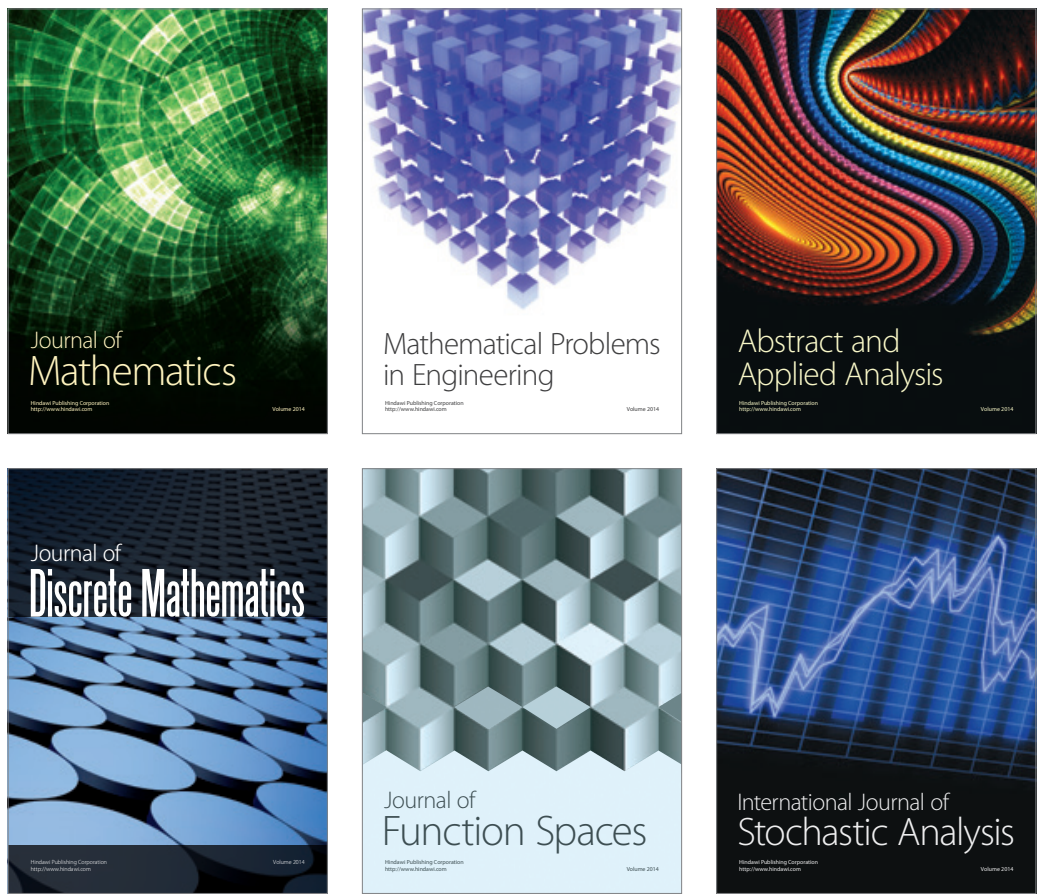

Journal of

Function Spaces

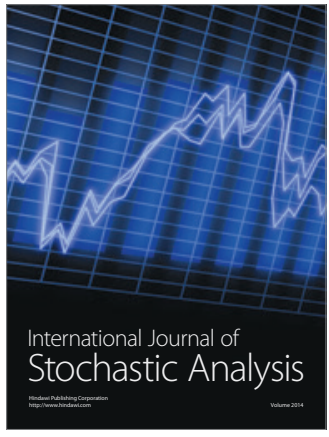

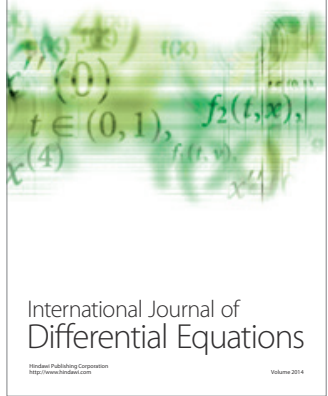
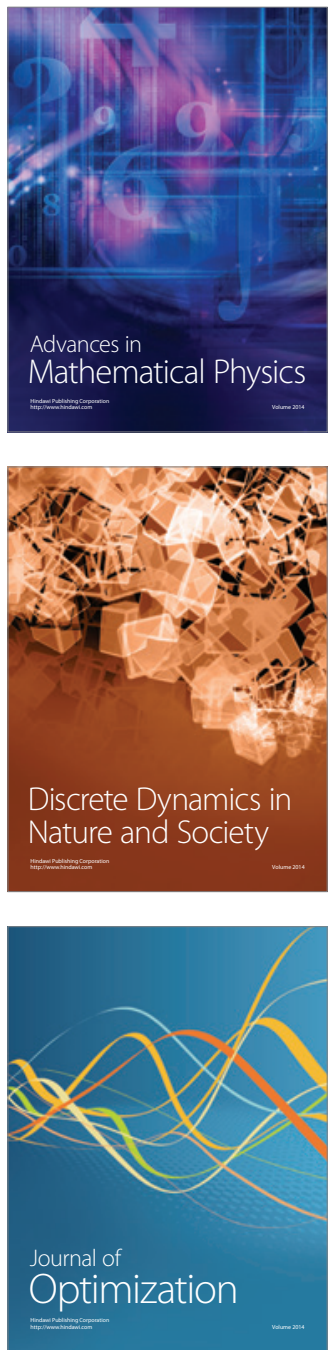